\title{
Self Control Deficits and Pathological Gambling
}

\author{
Karin Tochkov \\ Department of Psychology and Special Education, Texas A\&M University - Commerce \\ P.O. Box 3011, Commerce, TX 75429, USA \\ Tel: 1-903-468-6056 E-mail: Karin_Tochkov@tamu-commerce.edu
}

\begin{abstract}
The present paper focuses on the concepts of self-control and impulsivity as central features of clinical disorders like pathological gambling. The main purpose is to show the link between models in biopsychology and experiments with animals concerning self-control and models in clinical psychology involving patients with impulse control deficits. A representative model of each of the two research fields is discussed along with empirical studies and their implications for theory and treatment of pathological gambling.
\end{abstract}

Keywords: Self-control, Impulsivity, Pathological gambling

\section{Self-control deficits as the key link}

Self-control is defined as the ability to control one's impulses. The lack of self-control is called impulsivity. Individuals with disorders like pathological gambling, sex addiction and substance abuse have all in common that they have self-control deficits and are thus impulsive. One of the most used measures of self-control deficits in clinical studies is the discounting rate of delayed rewards. Since self-control deficits are signs of impulsivity and impatience to satisfy one's desires without delay, this measure provides a useful tool for assessment and therapy of pathological gamblers and substance abusers. Given the choice between an immediate reward and a larger reward at a future point of time, an impulsive individual would choose the smaller but immediate reward over the larger delayed reward, because of the impatience and the self-control deficit. This result implies a higher discounting rate for the impulsive individual.

But self-control and impulsivity are not restricted to the area of clinical psychology. These concepts were first developed in biopsychological studies and applied in animal experiments. Similar to the human participants in the clinical studies later, the animals were confronted with a choice between two alternatives: an immediate and a delayed. The measurement of self-control versus impulsivity resembled very much that of delayed discounting later. The evidence from these experiments suggests that, in general, animals have a very high rate of discounting and are thus highly impulsive.

In summary, the concepts of self-control and impulsivity can be interpreted as a key link between biopsychological experiments with animals and clinical studies with pathological gamblers and substance abusers. The former pioneered the research in the field and the latter applied the concepts to humans were able to analyze their findings based on the principles established by animal studies. The main purpose of the current study is to present the conceptual interrelation between animal learning and pathological gambling as shown in the application and meaning of self-control and impulsivity.

The present study is structured as following. At first, the biopsychological models of self-control and its measurement are discussed using one representative model (Rachlin \& Green, 1972). Then the clinical models of self-control deficits are presented with special emphasis on pathological gambling. In addition, the model of delayed discounting widely used in clinical studies is summarized. Finally, the implications of the concepts of self-control and impulsivity for the clinical research and practice are explored.

\section{The Biopsychological Model of Self-Control}

One of the most productive researchers in this area or psychology has been Rachlin. Despite a considerable amount of current research on the topic, the famous study of Rachlin and Green (1972) still serves as the basis for all future studies. Therefore, in the following this model is going to be discussed in more detail as representative for the biopsychological approach to self-control and impulsivity.

In this experiment, pigeons were confronted with two keys. The keys were dark for a certain amount of time ( $\mathrm{T}$ seconds) and then lit red and green respectively. A peck on the red light immediately produced 2 seconds of food; a peck on the green light produced 4 seconds of food but only after a delay of 4 seconds. After this the trial was 
repeated again. The red light represented an immediate small reinforcer; the green light represented a delayed large reinforcer. If the pigeon is impulsive and thus lacks self-control, then it is impatient and pecks the red light to receive the small immediate amount of food. If the pigeon exhibits more self-control and less impulsivity, then it pecks the green light to receive the large delayed amount of food. Confronted with the choice between the red and the green light, the pigeon almost invariably pecked the red light and produced the small immediate reinforcer. This result suggests that animals in general are impulsive.

A manipulation of the experimental setting produced additional results. At first a learning procedure was integrated. The pigeon was confronted with two keys that were dark for a certain amount of time (T seconds). Then only the green key was lit. The pigeon necessarily pecked the green light and produced the large delayed reinforcer. The purpose of this procedure was for the pigeon to learn to wait for a later but larger reinforcer, to be more patient and less impulsive.

Moreover, manipulation of the time until the keys are lit ( $\mathrm{T}$ seconds) resulted in a reversal of preferences. The pigeon had to wait different periods of time until the keys were lit and it could choose between red and green. When the time before the keys were lit was shorter, the pigeon was more likely to peck on the red light and receive the small immediate reinforcer. In this case it was more impulsive. When the time before the keys were lit was longer, the pigeon was more likely to peck the green light and receive the large delayed reinforcer. In this case it had more self-control and was less impulsive.

What was the reason for this reversal of preference? Suppose there are two cases: in the first the time before the keys were lit was short (1 second), in the second it was long (10 seconds). In the first case, the pigeon has to wait one second for the lights to be lit, and if it pecks the red key, the total time of waiting for the small immediate amount of food was one second. If the pigeon pecks the green key, then it had to wait 1 second plus it still has to wait for 4 more seconds to get the large delayed amount of food. Total time of waiting in this case is 5 seconds. A comparison between the total waiting time for the small and for the large reinforcer reveals that the pigeon has to wait five times longer to get the larger amount of food. This relatively long amount of waiting time causes the pigeon to be more impulsive and choose the small immediate reinforcer with a higher probability.

In the second case, the pigeon has to wait a total of 10 seconds to get the small immediate reinforcer and 14 seconds to get the large delayed one. The additional time of waiting for more food is 4 seconds, which is less than half of the time waiting for the small immediate reinforcer. In this case, the pigeon has to wait relatively less for a large delayed reinforcer than in case one. Therefore, it is more likely that the pigeon would be less impulsive and peck the green key.

The degree of self control in this experiment was measured as the relative rate of pecking on the red key. The formula for this measure, $\mathrm{R} / \mathrm{R}+\mathrm{G}$, consists of the number of pecks on the red key $(\mathrm{R})$ divided by the total amount of pecks on the red $(\mathrm{R})$ and green $(\mathrm{G})$ keys. If the relative rate of pecking on the red key is high, then the pigeon chooses more often the small immediate reinforcer and is therefore more impulsive. The lower the relative rate of pecking on the red key, the more often the green key is chosen and thus self-control is more likely to occur. The relative rate of pecking the red key is shown in Fig. 1 below as a function of the time before the keys are lit. The decreasing curve represents the main result of Rachlin and Green's study that the longer the waiting time before being able to choose between alternatives, the higher the probability of self-control behavior.

After Rachlin and Green (1972), many similar studies followed. Ainslie (1974) and Ainslie (1975) conducted also experiments with pigeons and obtained further evidence for the results of Rachlin and Green (1972). Mazur and Logue (1978) proposed another procedure of training the pigeons to choose the large delayed reinforcer. The delay for the small and for the large reinforcer was initially identical, but in consecutive trials, the delay for the smaller reinforcer was reduced and finally also eliminated.

\section{Clinical model of Self-Control}

In clinical psychology, self-control deficits are found in several disorders. The following discussion focuses on a representative model of pathological gambling developed by Petry (2001c). The study examines self-control and impulsivity in three groups of participants - pathological gamblers, pathological gamblers with substance use disorders and control group. The main question here was similar to the one of the Rachlin and Green's (1972) study with pigeons: Are pathological gamblers more impulsive or do they show more self-control than the control group? Furthermore, the additional influence of substance abuse on self-control was tested.

The participants in the study were asked to perform a simple behavioral task. Like the pigeons in Rachlin and Green's (1972) experiment, they had to choose between two alternatives: a smaller immediate money reward or a bigger delayed money reward. For this purpose they were asked questions like: "What do you prefer? $\$ 100$ 
now or $\$ 1000$ in one month?”. The delayed reward was always $\$ 1000$. The amount of the immediate reward was manipulated and increased in different trials with the delay time kept fixed. Then the delay time was allowed to vary and the amount of the immediate reward was kept fixed.

The study uses the rate of delayed discounting as a measure of self-control and impulsivity. The rate of discounting was used long ago and is used widely today, especially in business to measure the value of a money investment that delivers interest income at a future point of time. In psychology, Mazur (1987) adjusted the usual formula used in business to account for self-control deficits. The formula is represented as follows:

$$
\mathrm{DV}=\mathrm{V} /(1+\mathrm{kd})
$$

Where "DV" is the discounted value, i.e. today's value of the delayed $\$ 1000$ reward. "V" is the value of the delayed reward (here \$1000). "d" represents the delay time and "k" the degree of discounting as a measure of self-control deficit. The formula resembles very much the one used in Rachlin and Green (1972), where the relative pecks on the red light served as the measure for impulsiveness.

A simple example could clarify the role "k" plays in this framework. Suppose that two individuals participate in the study: one is impulsive (like a pathological gambler) and the other has self-control (like the control group). Since "k" is the measure of self-control deficit, the impulsive person would have a high "k", e.g. 0.1. The person with self-control would have low "k", e.g. 0.02. Assume that the delayed reward for both participants is $\$ 1000$ and the delay time one month. After substituting these values in the formula for delayed discounting, it comes out that the impulsive person values the $\$ 1000$ at $\$ 909$ in terms of today, the self-controlled person values the $\$ 1000$ higher at $\$ 980$. This results shows that the more impulsive a person is (the higher " $k ")$, the higher the rate of discounting and thus the less the value of delayed rewards in today's terms. This explains the behavior of an impulsive person like a pathological gambler, who would rather get a smaller immediate reward, because the value of delayed rewards is too low when discounted in the present moment. Therefore, as a more impulsive person a pathological gambler would be expected to have higher rate of discounting.

The results from the study of Petry (2001c) confirm this hypothesis and are represented in the diagram below.

Pathological gamblers were more impulsive and had a higher rate of delayed discounting than the control group. On the diagram, at every point of time the gamblers valued delayed rewards less than the control group. The pathological gamblers with alcohol addiction had even less self-control than the gamblers. Therefore this result suggests that an additional substance abuse disorder increases the rate of delayed discounting of gamblers.

Several other studies using the rate of delayed discounting as measurement of impulsivity have shown that patients with other types of addictions have also high discounting rates of delayed rewards and thus are more likely to act impulsively. Petry (2001a) and Petry (2001b) conducted experiments with different types of alcohol addicts and pathological gamblers. Bickel et al. (1999) focused on persons with different degree of cigarette smoking patterns. The study found out that the more intensive the smoking behavior, the higher the rate of delayed discounting. Also, discounting was bigger for delayed rewards offered in terms of cigarettes than for monetary rewards.

\section{Implications}

The two representative models discussed above along with the empirical studies have several major implications. The results of the clinical studies with humans reveal a broad consistence with the findings of the animal experiments, which confirms the importance for clinical patients of principles developed in animal studies and their applicability in clinical settings.

Moreover, clinical studies using the rate of delayed discounting as a measure of impulsivity suggest that rapid discounting of delayed rewards is a central feature of self-control deficits, like pathological gambling. This result enables clinicians to improve their analysis, treatment and prevention of self-control deficits.

The conclusion that pathological gamblers have higher rates of delayed discounting also opens the way for further research in the field, for example with the question of causality between discounting rates and self-control deficits, i.e. whether high discounting rates precede or stem from impulsive behavior.

\section{References}

Ainslie, G. W. (1974). Impulse control in pigeons. Journal of the Experimental Analysis of Behavior, 21, 485-489.

Ainslie, G. W. (1975). Specious reward: A behavioral theory of impulsiveness and impulse control. Psychological Bulletin, 82, 463-496. 
Bickel, W.K., Odum, A.L., \& Madden, G.J. (1999). Impulsivity and cigarette smoking: Delayed discounting in current, never, and ex-smokers. Psychopharmacology, 146, 447-454.

Mazur, J. E. (1987). An adjusting procedure for studying delayed reinforcement. In M.L. Commons, J. E. Mazur, J.A. Nevin \& H. Rachlin (Eds.), Quantitative analysis of behavior: Vol. 5. The effect of delay and of intervening events on reinforcement value (pp. 55-73). Hillsdale, NJ: Erlbaum.

Petry, N. M. (2001a). Delay discounting of money and alcohol in actively using alcoholics, currently abstinent alcoholics, and controls. Psychopharmacology, 154, 243-250.

Petry, N. M. (2001b). Substance abuse, pathological gambling and impulsivity. Drug and Alcohol Dependence, 63, 29-38.

Petry, N. M. (2001c). Pathological gamblers, with and without substance use disorders, discount delayed rewards at high rates. Journal of Abnormal Psychology, 110 (3), 482-487.

Rachlin, H., \& Green, L. (1972). Commitment, choice and self-control. Journal of Experimental Analysis of Behavior, 17, 15-22.

Siegel, E., \& Rachlin, H. (1995). Soft commitment: Self-control achieved by response persistence. Journal of Experimental Analysis of Behavior, 64, 117-128.

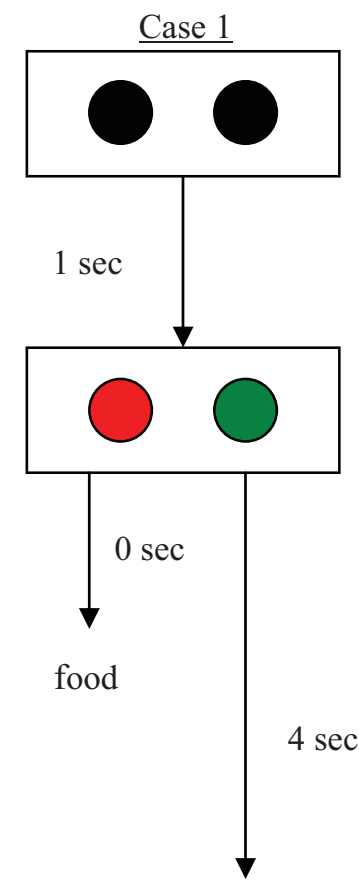

More food

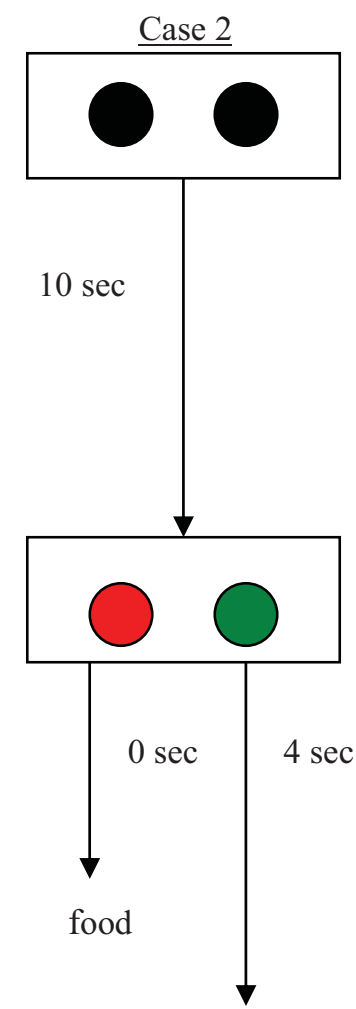

More food

Figure 1. Rachlin and Green (1972): The pigeon experiment with manipulation of the time until keys were lit 


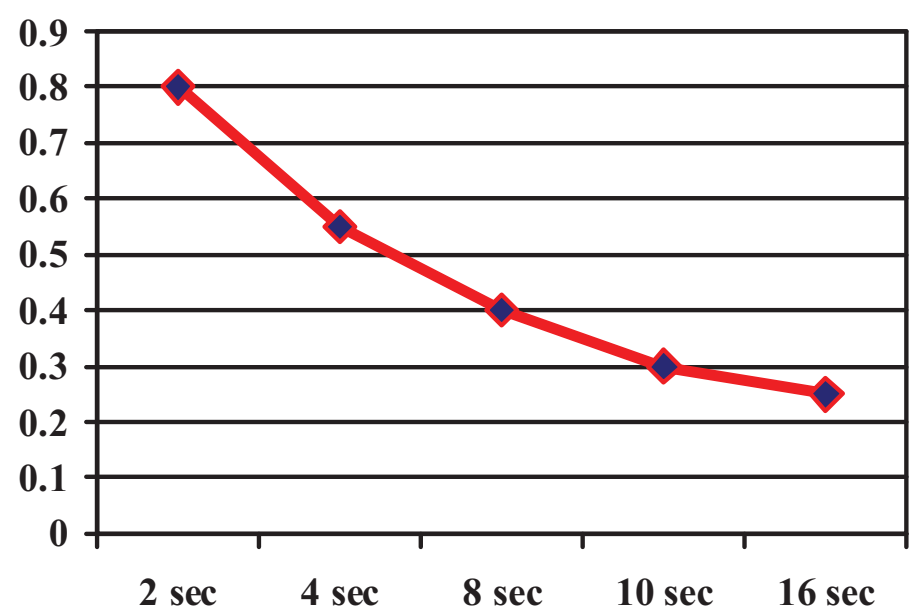

Figure 2. Rachlin and Green (1972): The relative rate of pecking the red key $(R / R+G)$ as a function of the time before the keys are lit

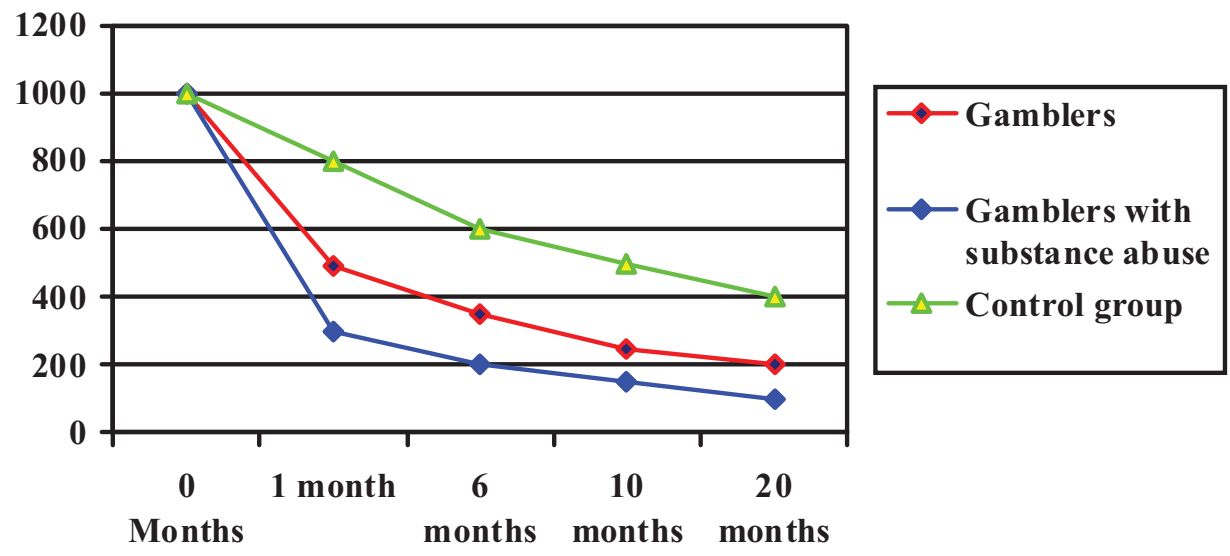

Figure 3. Petry (2001c): The discounted value of a delayed reward (\$1000) as a function of delay time for the three groups of participants in the study 